\title{
LDL/HDL ratio association with out-patient NIHSS score and dyslipidemic drug intake status as metabolic syndrome criteria of ischemic stroke patients at Dr. Sardjito General Hospital, Yogyakarta, Indonesia
}

\author{
Felicia Elberta ${ }^{1}$, Abdul Ghofir ${ }^{2}$, Imam Rusdi ${ }^{2}$ \\ ${ }^{1}$ Student of Medical Faculty Universitas Gadjah Mada, ${ }^{2}$ Neurology Departments of \\ Medical Faculty Universitas Gadjah Mada / Dr.Sardjito General Hospital, Yogyakarta, \\ Indonesia
}

DOI: http://dx.doi.org/10.19106/JMedScieSup005001201804

\section{ABSTRACT}

The LDL-C/HDL-C ratio (numeric) is a more significant predictor of IMT progression than LDL-C or HDL-C alone. The Objective is to check whether dyslipidemic or metabolic syndrome demonstrated by this ratio, affecting the NIHSS results data obtained to measure functional outcome. The data was analyzed using Pearson Chi-square with contingency table post-hoc analysis and Spearmann's Correlation with additional simplelinear regression. Out of 189 subjects, only 156 data are complete and valid. 70 patients were dyslipidemic, 65 were non-dyslipidemic, and the rest were unknown. There was a significant association of LDL/HDL ratio cut off point above 2.3 with dyslipidemic drug intake likely to produce a mild NIHSS outcome category (z score 2.1) (calculator is $\mathrm{P}$ value $=0.035729$ ), but not with other NIHSS categories. There was significant association of patients that did not take dyslipidemic drug with whatever LDL/HDL cut off point to the predictor outcome of Mild NIHSS category, but not with other NIHSS categories. The correlations were also insignificant between the LDL/HDL ratio and NIHSS score in one tailed $(p<0.36)$ and at two tailed $(p<0.72)$. Moreover, simple linear regression reveal that LDL/HDL ratio predicts $16 \%$ of NIHSS score, while taking anti dyslipidemic drugs which mean the person was dyslipidemic, and LDL/HDL ratio predict $31 \%$ of NIHSS outcome. Therefore LDL/HDL ratio had weak predictor value to NIHSS outcome, should not be an independent predictor.

Keywords: HDL/LDL ratio - NIHSS - ischemic stroke - cut off point - dyslipidemic drug status

*corresponding author: felicia.elberta.w@mail.ugm.ac.id 